\title{
Functional outcome of arthroscopic Bankart repair in post-traumatic recurrent shoulder dislocation in a private tertiary care teaching hospital in eastern India
}

\author{
Ayon Das ${ }^{1}$, Subhrojyoti Bhowmick ${ }^{2}$, Nikhilesh Das ${ }^{1}$, Swarnendu Samanta ${ }^{1}$, \\ Somnath De ${ }^{1}$, Sayan Das ${ }^{3 *}$
}

\begin{abstract}
${ }^{1}$ Department of Orthopaedics, Peerless Hospital \& B.K. Roy Research Centre, Kolkata, West Bengal, India ${ }^{2}$ Department of Academics, Quality \& Research, Peerless Hospital \& B.K. Roy Research Centre,

Kolkata, West Bengal, India

${ }^{3}$ Department of Pharmacology, Dr. D.Y. Patil Medical College, Hospital \& Research Centre, Pune, Maharashtra, India
\end{abstract}

Received: 01 August 2020

Accepted: 16 September 2020

\section{*Correspondence:}

Dr. Sayan Das,

E-mail: doc.sayan.das@gmail.com

Copyright: (C) the author(s), publisher and licensee Medip Academy. This is an open-access article distributed under the terms of the Creative Commons Attribution Non-Commercial License, which permits unrestricted non-commercial use, distribution, and reproduction in any medium, provided the original work is properly cited.

\section{ABSTRACT}

Background: Recurrent anterior shoulder dislocations are quite common in young patients with Bankart lesions. The open Bankart repair was the gold standard for years, however arthroscopic Bankart repair has gained popularity. The aim of the study was to evaluate the functional results of arthroscopic Bankart repair in patients with post-traumatic recurrent anterior glenohumeral instability.

Methods: Patients who underwent arthroscopic Bankart repair for post-traumatic recurrent anterior shoulder dislocation were included in the study. This was a prospective study conducted at a tertiary care Hospital in Kolkata, India between August 2017 to May 2019. All patients were followed up at an interval of 2 weeks, 6 weeks, 12 weeks, 6 months, 9 months and 1 year. Rowe score was used to assess the functional outcomes of the patients.

Results: 34 (91.9\%) patients reported good to excellent results, whereas only $2(5.4 \%)$ patients had fair outcome and 1 $(2.7 \%)$ patient demonstrated poor surgical result. The total rowe score improved from 46.62 pre-operatively to 94.20 post-operatively after 1 year $(\mathrm{p}<0.01)$. By the end of 1 -year follow-up, $35(94.6 \%)$ patients had full range of motion in all the planes. Majority of (89.2\%) patients did not have any complications. Only 1 patient out of 37 had a recurrence $(2.7 \%)$.

Conclusion: Arthroscopic Bankart repair is an effective, cosmetic and safe surgical procedure with good clinical outcomes and excellent post-operative shoulder range of motion. This surgical intervention also has low recurrence rate and minimum surgical complications.

Keywords: Bankart repair, Arthroscopy, Shoulder dislocation, Shoulder surgery, Recurrent shoulder injury, Shoulder instability

\section{INTRODUCTION}

The shoulder is the most commonly dislocated joint in the body, because of its relative lack of bony limitations and extensive range of motion. ${ }^{1}$ The glenohumeral joint requires a stable pivot for keeping the upper extremity at a wider arc of a circle in the three-dimensional plane. The special orientation of the shoulder provides the glenohumeral joint with a high degree of mobility at the cost of stability. 
The glenohumeral joint has the most extensive range of movement as compared to any joint in the body. It is one of the most active joints in the body and because of its role in the protective reflex of the body, it is often injured. Shoulder dislocations comprise approximately $50 \%$ of all joint dislocations. The stability of the shoulder joint depends on static and dynamic soft tissue structures such as the labrum, the glenohumeral ligaments, and the rotator cuff. $^{2}$ Dislocation of the shoulder joint occurs in 1 to $2 \%$ of the population. ${ }^{3}$ Its incidence is $1.7 \%$ among adults and it is three times more common among men. ${ }^{4} 90 \%$ of shoulder dislocations are anterior, and traumatic injuries account for $95 \%$ of them. ${ }^{5,6}$ In athletic patients under the age of 20 years, the recurrence rates are greater than $90 \%$ whereas in patients aged between 20 to 25 years, the rates are between 50 and $75 \% .^{6} \mathrm{~A}$ family history of instability is found in almost $25 \%$ of patients who sustain a shoulder dislocation. $^{7}$

Main complication of shoulder dislocation is recurrent instability and it accounts for an average of $70-90 \%$ recurrence rate in patients between 20-40 years of age. Following dislocation, injuries occur to surrounding structure such as bone, cartilage and soft tissues, resulting in various pathological conditions. During shoulder dislocations, humeral head is forced anteriorly out of glenoid cavity resulting in detaching fibrocartilaginous labrum from the anterior rim of glenoid cavity. This detachment of glenoid labrum is called Bankart lesion. Bankart lesion is the most common and essential lesion in treatment of anterior shoulder instability. ${ }^{8}$

Management of recurrent anterior shoulder dislocation is by reattachment of labro-ligamentous structure to the glenoid. Bankart repair techniques have improved significantly over the last 90 years since Bankart initially described this lesion. The open Bankart repair was the gold standard for years, with success rates ranging from $75 \%$ to $100 \%$; however, postoperative problems such as restriction to external rotation and secondary osteoarthritis were concerns. ${ }^{9,10}$ Arthroscopic Bankart repair gained popularity since it began almost 30 years ago because of improved arthroscopic equipment and increased experience of surgeons. ${ }^{11}$ Recent advancements in the techniques, suture material, bioabsorbable anchors and concomitant treatment of other pathologies, arthroscopic repair has become a standard care. ${ }^{12}$

\section{METHODS}

Institutional ethics committee (IEC) approval was taken before the start of the study. Initially, 39 patients with post traumatic recurrent anterior shoulder dislocation who were treated by arthroscopic Bankart repair in Peerless Hospital and B. K. Roy Research Centre, Kolkata from August 2017 to May 2019 and fulfilling the inclusion criteria were enlisted in this study. But among them 1 patient suffered a proximal humerus fracture on the same arm due to road traffic accident after 5 weeks of surgery and 1 patient lost follow-up immediately after suture removal. So, 37 patients with a minimum 1 year of follow-up were finally considered in this study.

\section{Inclusion criteria}

The study subjects were included based on the following inclusion criteria: patients above 18 years of age who are skeletally mature, sustained a post-traumatic anterior dislocation of shoulder of more than 1 episode, Bankart lesion confirmed by Magnetic resonance imaging (MRI).

\section{Exclusion criteria}

The subjects were excluded from the study based on the following exclusion criteria: other shoulder pathologies such as bony Bankart, revision Bankart, Hill-Sachs lesion involving more than $25 \%$ of the humeral head, habitual dislocations, rotator cuff tear, SLAP lesion, multidirectional instability or posterior instability of shoulder, arthritis of shoulder, past history of fracture of upper end of humerus or operated earlier for any shoulder problem and patients with associated neuromuscular disorder or weakness or seizure disorder or any other mental illness.

\section{Pre-operative assessment}

The patients were subjected to a thorough history, clinical examination and pre-operative routine laboratory investigations, which was supplemented by radiographs in antero-posterior, lateral, axillary and scapular $\mathrm{Y}$ view along with MRI scan of the affected shoulder joint.

\section{Operative procedure}

\section{Anaesthesia and positioning}

All the surgical procedures were performed under general anesthesia with the patients in lateral decubitus position. An arm holder was used to give traction as well as to keep the shoulder in 45 degrees abduction and 20 degrees forward flexion.

\section{Portal placement and diagnostic arthroscopy}

The primary posterior portal was created $1.5 \mathrm{~cm}$ inferior and $1.5 \mathrm{~cm}$ medial to the posterolateral edge of the acromion. A complete evaluation of the capsular complex and osseous structures was done. The Bankart lesion was identified, confirmed and probed. Antero-superior and antero-central portals were created in outside-in fashion using spinal needle localization.

\section{Glenoid preparation}

The labral tissue was elevated medially to free up the labrum from anterior glenoid with the help of angled elevator. Rasping of glenoid neck was done to prepare the redundant capsular tissue prior to plication. 


\section{Anchor placement and capsulolabral plication}

3 anchors preloaded with fibre-wires were put in after making pilot holes at 1,3 and 5 o'clock for right shoulder and 7,9 and 11 o'clock for left shoulder. The inferior most anchors (5 o'clock for right and 7 o'clock for left) were placed first.

\section{Knot tying}

Capsular stitches were taken 1 o'clock below the respective anchor using suture shuttle and relayed using crochet hook. Then the capsular tissue was secured with six alternate half stitches using a simple knot pusher. At the end of the procedure, it was confirmed whether the capsule-labral complex was like a 'bump' at the glenoid margin and the humeral head was centrally placed.

\section{Skin closure and shoulder immobilization}

Non-absorbable suture was used to close the skin. Sterile dressings were applied to the surgical incisions. Patients were placed in a shoulder immobilizer.

\section{RESULTS}

\section{Age distribution}

The mean age in this study was 26.16 years. The youngest patient was 19 years old and the oldest patient was 42 years old. Overall, $83.8 \%$ patients were between the age group of 20-40 years. It might be because younger peoples are more active and involved in outdoor and sports activities which makes them more prone to injuries.

\section{Table 1: Age distribution.}

\begin{tabular}{|c|c|c|c|}
\hline $\begin{array}{l}\text { Age } \\
\text { (years) }\end{array}$ & $\begin{array}{l}\text { No. of } \\
\text { patients }\end{array}$ & $\begin{array}{l}\text { Percentage } \\
(\%)\end{array}$ & $P$ value \\
\hline $18-20$ & 5 & 13.5 & \multirow{5}{*}{0.001} \\
\hline $20-30$ & 25 & 67.6 & \\
\hline $30-40$ & 6 & 16.2 & \\
\hline$>40$ & 1 & 2.7 & \\
\hline Total & 37 & 100.0 & \\
\hline
\end{tabular}

\section{Sex distribution}

In this study, 33 patients $(89.2 \%)$ were male and 4 patients were female $(10.8 \%)$. Majority of patients were male $(89.2 \%)$ which may be because of more outdoor and sports related activities makes them more vulnerable to accidents and trauma.

\section{Side of injury distribution}

In this study, number of right shoulder involvement was $28(75.7 \%)$ which was much higher in comparison with left shoulder involvement which was 9 (24.3\%). Higher incidence of right shoulder involvement (75.7\%) may be due to right hand dominance.

\section{Occupation distribution}

In this study, $20(54.1 \%)$ patients were involved in significant occupation requiring high demand or overhead activities such as students with sports activities, farmers, teacher and professional sportsmen.

\section{Pre-operative dislocations distribution}

Among 37 patients, 1 (2.7\%) patients had $\geq 10$ episodes of dislocation. 4 (10.8\%) patients had 6 to 9 episodes of dislocation of the shoulder in the preceding year, and 32 $(86.5 \%)$ patients suffered 1 to 4 episodes in the preceding year pre-operatively. The average no. of pre-operative dislocations was 3.62.

Table 2: Pre-operative dislocations distribution.

\begin{tabular}{|llll|}
\hline $\begin{array}{l}\text { Total no. of } \\
\text { dislocations }\end{array}$ & $\begin{array}{l}\text { No. of } \\
\text { patients }\end{array}$ & $\begin{array}{l}\text { Percentage } \\
(\%)\end{array}$ & P value \\
\hline $\mathbf{1 - 5}$ & 32 & 86.5 & \\
\hline $\mathbf{6 - 9}$ & 4 & 10.8 & \\
\hdashline $\mathbf{1 0}$ & 1 & 2.7 & \multirow{2}{*}{0.001} \\
\hline Total & 37 & 100.0 & \\
\hline
\end{tabular}

\section{Total Rowe score}

The total Rowe score (mean and SD) improved from $46.62 \pm 23.24$ pre-operatively to $94.20 \pm 15.92$ postoperatively in the final follow-up at the end of 1 year and $\mathrm{Z}$ value was found to be -5.174. A dip in the total Rowe score at 2 weeks was due to the shoulder immobilization post-surgery.

Table 3: Total Rowe score.

\begin{tabular}{|l|l|}
\hline Total Rowe score & Mean score \\
\hline Pre-operative & 46.62 \\
\hline Post-operative & \\
\hline 2 weeks & 35 \\
\hline 6 weeks & 71.22 \\
\hline 12 weeks & 73.38 \\
\hline 6 months & 89.86 \\
\hline months & 93.51 \\
\hline 1 year & 94.20 \\
\hline
\end{tabular}

\section{Final Rowe outcome}

In a total of 37 patients, $32(86.5 \%)$ patients showed excellent and $2(5.4 \%)$ patients had good results at the end of 1-year follow-up, whereas $2(5.4 \%)$ patients had fair outcome and $1(2.7 \%)$ patient demonstrated poor surgical result. 


\section{Complications}

Out of 37 patients, $33(89.2 \%)$ patients did not have any complications, whereas $2(5.4 \%)$ patients had shoulder stiffness, $1(2.7 \%)$ patient complained of mild pain and 1 $(2.7 \%)$ patient had a re-dislocation episode postoperatively.

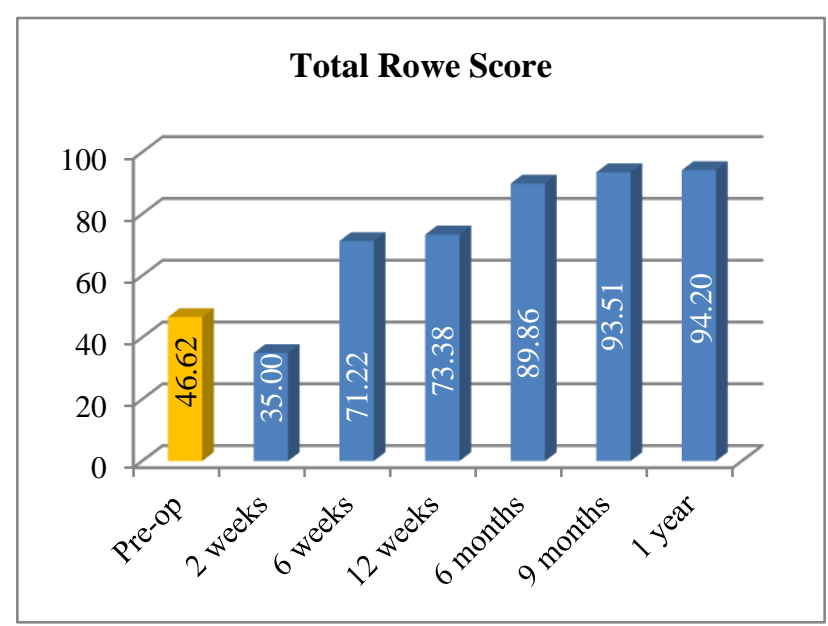

Figure 1: Total Rowe score.

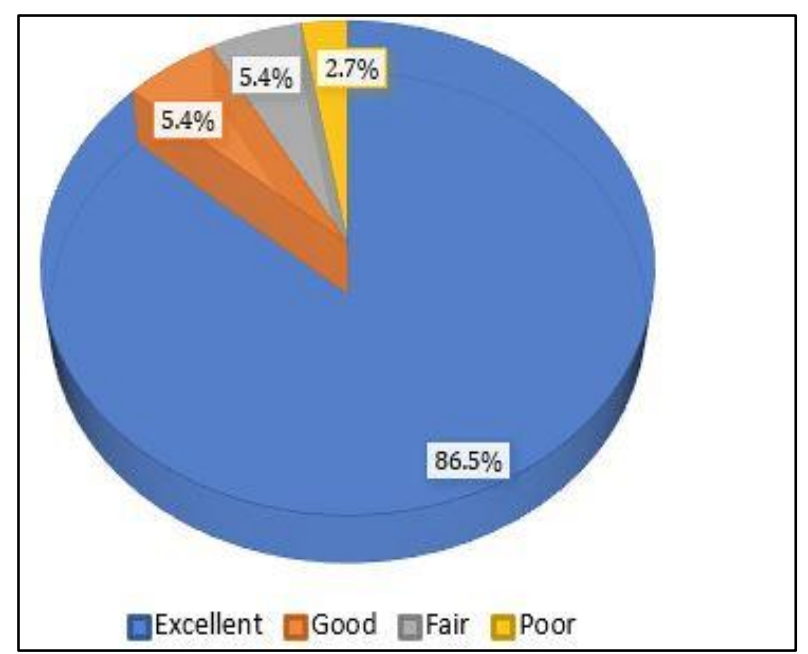

Figure 2: Final Rowe score.

Majority of patients had symptoms for a period ranging from 1-5 years $(78.4 \%)$. All patients had a positive apprehension test before surgery. $33(89.2 \%)$ patients had limitation of external rotation on 90 degrees abduction preoperatively. 3 suture anchors were used intra-operatively in all patients. There were no intraoperative complications related to the arthroscopic procedure with regard to nerve injuries, compartment syndrome, infection or anchor loosening. The mean post-operative shoulder score significantly improved at the time of the final follow-up. The total Rowe score improved from a mean and standard deviation (SD) of $46.62 \pm 23.24$ pre-operatively to $94.20 \pm 15.92$ post-operatively. 35 patients achieved full range of shoulder movement by the end of 1 year.
Table 4: Final Rowe outcome.

\begin{tabular}{|c|c|c|c|}
\hline $\begin{array}{l}\text { Final Rowe } \\
\text { Outcome }\end{array}$ & $\begin{array}{l}\text { No. of } \\
\text { Patients }\end{array}$ & $\begin{array}{l}\text { Percentage } \\
(\%)\end{array}$ & $P$ value \\
\hline Excellent & 32 & 86.5 & \multirow{5}{*}{0.001} \\
\hline Good & 2 & 5.4 & \\
\hline Fair & 2 & 5.4 & \\
\hline Poor & 1 & 2.7 & \\
\hline Total & 37 & 100.0 & \\
\hline
\end{tabular}

Table 5: Complications.

\begin{tabular}{|c|c|c|c|}
\hline Complication & $\begin{array}{l}\text { No. of } \\
\text { Patients }\end{array}$ & $\begin{array}{l}\text { Percentage } \\
(\%)\end{array}$ & $P$ value \\
\hline Stiffness & 2 & 5.4 & \multirow{5}{*}{0.001} \\
\hline Pain & 1 & 2.7 & \\
\hline Re-dislocation & 1 & 2.7 & \\
\hline None & 33 & 89.2 & \\
\hline Total & 37 & 100.0 & \\
\hline
\end{tabular}

\section{DISCUSSION}

Shoulder, by virtue of its anatomy and biomechanics, is one of the most unstable and frequently dislocated joints in the body. The Bankart lesion represents the most common form of labro-ligamentous injury in patients with traumatic anterior dislocation of the shoulder. Surgical management is by reattachment of the labro-ligamentous complex to the glenoid either arthroscopically or by an open procedure (Bankart repair).

When treating shoulder instability, the ideal technique should include the ability to assess the glenohumeral joint instability with regard to the type of lesion, the anatomic structures involved, its potential for healing and the type of fixation needed. ${ }^{12}$ The ideal technique should also avoid injuries to the surrounding normal tissues. Unlike the open method of Bankart repair, which renders significant loss of range of motion because of disruption of the subscapularis tendon, the shoulder arthroscopic method creates minimal tissue trauma. ${ }^{14-16}$

Rowe score was used in this study because it is widely used with much historic significance as well as its simplicity of application on Outpatient department (OPD) basis by the examining clinician, which makes it widely used for evaluation acceptable. In this study, all the patients were young and physically active, mostly males (89.2\%), age ranging from 19-42 years with mean age 26.16 years, with higher incidence of right-hand involvement $(75.7 \%)$ who were engaged in jobs of varying demand. Similar results were seen in studies conducted by Katti et al in Karnataka (India) which had a mean age of 27.19 years with $90.5 \%$ males and $70.8 \%$ right hand involvement, Jaju et al in Maharashtra (India) having mean age of 27.31 years with 98.3\% males and $70.8 \%$ right hand involvement, and Ramesh et al in Telangana (India) who had $90 \%$ males and $70 \%$ right hand involvement in the study. $8,17,18$ 
In this study, the average number of pre-operative dislocations was 3.62. This is corelating with studies done by Katti et al (3.1) and Jaju et al (3.96) ${ }^{8,17}$ All the patients (37 patients) had positive apprehension test response preoperatively. Studies conducted by Sedeek et al in Singapore, Erkoçak et al in Turkey and Kalkar et al in Turkey, all had pre-operative positive apprehension in $100 \%$ patients. ${ }^{19,20,21}$ The mean pre-operative total Rowe score in our study was 46.62 which is close to the studies conducted by Sahu et al and Ramesh et al which had a total Rowe score of 48.51 and 45.5 pre-operatively respectively. ${ }^{18,22}$

In all of the patients, 3 suture anchors were used. Boileau et al in France in a study concluded by suggesting at least four anchor points should be used to obtain secure shoulder stabilization. ${ }^{23}$ Shibata et al in Japan showed in their study that use of more than 3 anchors decreases the recurrence rate and prevents re-dislocation within the first year of surgery. ${ }^{24} 2$ patients post-operatively experienced apprehension in our study. Study by Mishra et al in New Delhi (India) had 2 and by Chandra et al had 1 patient with apprehension post-operatively. ${ }^{25,26}$

The mean post-operative total Rowe score in our study was 94.20 which is almost similar to studies performed by Kalkar et al, Jadhav et al and Parmar et al in having a total Rowe score of $95.5,95.63$ and 94.16 post-operatively respectively. ${ }^{21,27,28}$ In this study, out of 37 patients, 32 $(86.5 \%)$ came out with excellent results, $2(5.4 \%)$ showed good, $2(5.4 \%)$ showed fair and $1(2.7 \%)$ had poor outcome at the end of 1-year follow-up period. The results found were similar to the ones reported by Erkoçak et al, Sahu et al, Sood et al and Kalkar et al 1 out of the 37 patients included in the study experienced one episode of shoulder dislocation at 8 months post-surgery which occurred while lifting a heavy weight overhead..$^{20,21,22,29}$ Similar results of 1 episode of re-dislocation post operatively was seen in studies of Kalkar et al and Katti et al. ${ }^{8,21}$

Arthroscopic techniques described previously used transglenoid sutures or bioabsorbable tracks. In the past few years, newer techniques involving suture anchor fixation and capsular plication have evolved with promising results. Suture anchors are low-profile fixation devices that minimize articular surface damage of the humeral head offering anatomic reconstruction of the glenoid labrum as well as the glenohumeral ligament complex.

Arthroscopic treatment of shoulder instability has certain advantages compared to open procedures, which includes smaller skin incisions, more complete inspection of the glenohumeral joint with access to all areas for repair, shorter surgical time, less morbidity, less postoperative pain and narcotic use, reduced hospitalization time, faster postoperative recovery and rehabilitation and a decreased risk of complications with maximum preservation of external rotation. ${ }^{30}$
Limitations of our study include non-randomization, small group of patients, short follow-up period of 1 year and a lack of Control group.

\section{CONCLUSION}

Arthroscopic Bankart repair with suture anchors in cases of recurrent post-traumatic anterior shoulder instability is a reliable surgical procedure with respect to shoulder function and motion as it offers good clinical outcome, excellent post-operative shoulder range of motion with improved function. This surgical procedure has better patient compliance, low recurrence rate and minimum surgical complications in properly selected patients.

A larger well-designed study is required to be conducted across the country to corroborate the findings of our study.

Funding: No funding sources

Conflict of interest: None declared

Ethical approval: The study was approved by the institutional ethics committee

\section{REFERENCES}

1. Bankart AB. Recurrent or habitual dislocation of the shoulder-joint. British medical journal. 1923;2(3285): 1132.

2. Lippitt S, Matsen F. Mechanisms of glenohumeral joint stability. Clinical orthopaedics and related research. 1993;(291):20-8.

3. Kazár B, Relovszky E. Prognosis of primary dislocation of the shoulder. Acta Orthop Scand. 1969;40(2):216-24.

4. Hovelius L. Incidence of shoulder dislocation in Sweden. Clinical orthopaedics and related research. 1982;(166):127-31.

5. Goss TP. Anterior glenohumeral instability. Orthopedics. 1988;11(1):87-95.

6. Hovelius L. Anterior dislocation of the shoulder in teen-agers and young adults. Five-year prognosis. The Journal of bone and joint surgery. American volume. 1987;69(3):393-9.

7. Rowe CR. Acute and recurrent anterior dislocations of the shoulder. The Orthopedic clinics of North America. 1980;11(2):253.

8. Katti S, Ballal DH, Kumar H. Functional outcome of arthroscopic Bankart repair for anterior shoulder instability. Int J Orthop. 2017;3(1):267-71.

9. An VV, Sivakumar BS, Phan K, Trantalis J. A systematic review and meta-analysis of clinical and patient-reported outcomes following two procedures for recurrent traumatic anterior instability of the shoulder: Latarjet procedure vs. Bankart repair. Journal of shoulder and elbow surgery. 2016;25(5):853-63.

10. Owens BD, Cameron KL, Peck KY, DeBerardino TM, Nelson BJ, Taylor DC, et al. Arthroscopic versus open stabilization for anterior shoulder 
subluxations. Orthopaedic journal of sports medicine. 2015;3(1):2325967115571084.

11. Cole BJ, L'insalata J, Irrgang J, Warner JJ. Comparison of arthroscopic and open anterior shoulder stabilization: a two to six-year follow-up study. JBJS. 2000;82(8):1108.

12. Sperling JW, Smith AM, Cofield RH, Barnes S. Patient perceptions of open and arthroscopic shoulder surgery. Arthroscopy: The Journal of Arthroscopic \& Related Surgery. 2007;23(4):361-6.

13. Trenhaile SW, Savoie III FH. New frontiers in arthroscopic treatment of glenohumeral instability. Arthroscopy: The Journal of Arthroscopic \& Related Surgery. 2002;18(2):76-87.

14. Kim SH, Ha KI, Cho YB, Ryu BD, Oh I. Arthroscopic anterior stabilization of the shoulder: two to six-year follow-up. JBJS. 2003;85(8):1511-8.

15. Green MR, Christensen KP. Arthroscopic Bankart procedure: two-to five-year followup with clinical correlation to severity of glenoid labral lesion. The American journal of sports medicine. 1995; 23(3):276-81.

16. Ryu RK. Arthroscopic approach to traumatic anterior shoulder instability. Arthroscopy. 2003;19(10):94-101.

17. Jaju R, Aziz AM, Sadigale V. Functional results of arthroscopic bankart's Repair (ABR) in bankart's lesion of the shoulder. International Journal of Orthopaedics. 2018;4(1):1109-15.

18. Ramesh G, Kumar NR, Vardhan MV. A study of management of recurrent dislocation of shoulder by arthroscopic bankart's repair. Asian Pac. J. Health Sci. 2016;3(3):294-300.

19. Sedeek SM, Tey IK, Tan AH. Arthroscopic Bankart repair for traumatic anterior shoulder instability with the use of suture anchors. Singapore medical journal. 2008;49(9):676.

20. Erkoçak ÖF, Yel M. The Functional Results of Arthroscopic Bankart Repair with Knotless Anchors for Anterior Glenohumeral Instability. European Journal of General Medicine. 2010;7(2).

21. Kalkar İ, Esenyel CZ, Saygilı MS, Esenyel A, Gürbüz H. The results of Bankart repair without capsular plication in patients with recurrent traumatic anterior shoulder dislocation. Journal of Orthopaedic Surgery. 2017;25(1):2309499016684753.

22. Kumar S, Kumar A. Arthroscopic repair of bankart's lesion using suture anchors in recurrent anterior shoulder instability. Journal of Evidence Based Medicine and Healthcare. 2015;2(24):3514-28.

23. Boileau P, Villalba M, Héry JY, Balg F, Ahrens P, Neyton L. Risk factors for recurrence of shoulder instability after arthroscopic Bankart repair. JBJS. 2006;88(8):1755-63.

24. Shibata H, Gotoh M, Mitsui Y, Kai Y, Nakamura H, Kanazawa $\mathrm{T}$ et al. Risk factors for shoulder redislocation after arthroscopic Bankart repair. Journal of orthopaedic surgery and research. 2014;9(1):53.

25. Mishra A, Sharma P, Chaudhary D. Analysis of the functional results of arthroscopic Bankart repair in posttraumatic recurrent anterior dislocations of shoulder. Indian journal of orthopaedics. 2012;46(6):668.

26. Chandra M, Basak S, Bhar D, Mondal P, Halder PS, Das A. Evaluation of results of arthroscopic Bankart repair for post traumatic anterior shoulder instability. Journal of evolution of medical and dental sciencesjemds. 2015;4(66):11459-67.

27. Jadhav S, Deshpande S, Patil S. Arthroscopic evaluation and management of recurrent anterior shoulder instability. International journal of scientific research. 2018;6(4).

28. Parmar RS, Kapoor S, Sharma B. Arthroscopic Bankart repair following traumatic recurrent shoulder dislocation: A prospective review of 30 cases. Journal of Orthopaedic Surgery. 2019; 27(1):2309499019832708.

29. Sood M, Ghai A. Functional outcome after arthroscopic management of traumatic recurrent dislocation shoulder using Bankart repair and Remplissage techniques. Medical Journal Armed Forces India. 2018;74(1):51-6.

30. McIntyre LF, Caspari RB, Savoie III FH. The arthroscopic treatment of multidirectional shoulder instability: two-year results of a multiple suture technique. Arthroscopy: The Journal of Arthroscopic \& Related Surgery. 1997;13(4):418-25.a J Med Sci. 1999;48(3):99-100.

Cite this article as: Das A, Bhowmick S, Das N, Samanta S, De S, Das S. Functional outcome of arthroscopic Bankart repair in post-traumatic recurrent shoulder dislocation in a private tertiary care teaching hospital in eastern India Int J Res Orthop 2020;6:1233-8. 